\title{
Wavelength requirements for a scalable multicast single-hop WDM network
}

\begin{abstract}
In this paper, we present a method for designing a passive optical based single-hop wavelength division multiplexing multicast architecture that can achieve a scalable structure and form the basis of a wavelength efficient single-hop WDM network. The proposed architecture minimizes the number of wavelengths required for efficient multicast service and also minimizes tunability requirement of the transceivers. The network size scalability is achieved by adding transmitters and receivers to the designated groups. We show that the proposed system can accommodate large tuning delays and keeps with suitable throughput when the number of wavelength is equal to the number of nodes. We also show that the design can lead to a scalable structure while minimizing the number of wavelengths and tunability of the transceivers required for an efficient multicast service resulting in an improved system throughput and delay performance.
\end{abstract}

Keyword: Multicasting; Wavelength division multiplexing; Passive optical network 